Published in final edited form as: Graefes Arch Clin Exp Ophthalmol. 2010 October ; 248(10): 1377-1386. doi:10.1007/ s00417-010-1381-2.

\title{
Multimodal fundus imaging in Best vitelliform macular dystrophy
}

\author{
Daniela C. Ferrara, \\ Department of Ophthalmology, Otolaryngology and Head and Neck Surgery, Faculdade de \\ Medicina de Ribeirão Preto, Universidade de São Paulo, Ribeirão Preto, SP, Brazil
}

MACIMA - Macular Imaging and Treatment Division, Hospital de Olhos de Araraquara, Rua Padre Duarte 1786, Araraquara, SP 14.801-310, Brazil

\section{Rogério A. Costa,}

Department of Ophthalmology, Otolaryngology and Head and Neck Surgery, Faculdade de Medicina de Ribeirão Preto, Universidade de São Paulo, Ribeirão Preto, SP, Brazil

MACIMA - Macular Imaging and Treatment Division, Hospital de Olhos de Araraquara, Rua Padre Duarte 1786, Araraquara, SP 14.801-310, Brazil

CBCV - Centro Brasileiro de Ciências Visuais, Belo Horizonte, MG, Brazil

\section{Stephen Tsang,}

Department of Ophthalmology and Pathology, and Department of Ophthalmology and Cell Biology, Columbia University, New York, NY, USA

\section{Daniela Calucci,}

MACIMA - Macular Imaging and Treatment Division, Hospital de Olhos de Araraquara, Rua Padre Duarte 1786, Araraquara, SP 14.801-310, Brazil

CBCV - Centro Brasileiro de Ciências Visuais, Belo Horizonte, MG, Brazil

Rodrigo Jorge, and

Department of Ophthalmology, Otolaryngology and Head and Neck Surgery, Faculdade de Medicina de Ribeirão Preto, Universidade de São Paulo, Ribeirão Preto, SP, Brazil

\section{K. Bailey Freund}

Vitreous Retina Macula Consultants of New York, New York, NY, USA

Rogério A. Costa: roger.retina@globo.com

\section{Abstract}

Background-Best vitelliform macular dystrophy (BVMD) is a rare autosomal dominant retinal disease of highly variable phenotypic expression. Interpretations of disease mechanisms based on histopathology, electrophysiology, genetic analysis, and retinal imaging are somewhat discordant in fundamental issues such as the location and extension of primary retinal changes. Herein we describe the morphological macular features in patients with BVMD undergoing simultaneous multimodal fundus imaging and compare to those of normal age-matched subjects.

Methods-Comparative study including seven patients with BVMD (14 eyes) and seven agematched healthy subjects (14 eyes). All participants were submitted to complete ophthalmological 
examination, fundus photography, and standardized multimodal fundus imaging protocol including Fourier-domain optical coherence tomography (Fd-OCT) combined with near-infrared reflectance and blue-light fundus autofluorescence (FAF).

Results-In two eyes in the "subclinical" stage, Fd-OCT revealed thickening of the middle highly reflective layer (HRL) localized between the photoreceptors' inner/outer segments junction (inner-HRL) and RPE/Bruch's membrane reflective complex (outer-HRL) throughout the macula. In one eye in the "vitelliform" stage, a homogeneous hyper-reflective material on Fd-OCT was observed between the middle-HRL and outer-HRL; this material presented increased fluorescence on FAF. The outer nuclear layer (ONL) was thinned in the central macula and subretinal fluid was not identified in these earlier disease stages. In patients of "pseudohypopyon" (two eyes), "vitelliruptive" (eight eyes) and "atrophic" (one eye) stages, Fd-OCT revealed a variety of changes in the middle- and inner-HRLs and thinning of ONL. These changes were found to be associated with the level of visual acuity observed. Thickening of the middle-HRL was observed beyond the limits of the clinically evident macular lesion in all eyes.

Conclusions-Multimodal fundus imaging demonstrated thickening of the reflective layer corresponding to the photoreceptors' outer segments throughout the macula with no subretinal fluid accumulation as the earliest detectable feature in BVMD. Changes detected in the photoreceptors' reflective layers (middle- and inner- HRLs) and ONL thinning seemed to be progressive with direct implications for the level of visual acuity impairment observed among the different stages of the disease.

\section{Keywords}

Best disease; Fourier-domain; Fundus autofluorescence; Infrared; Retinal pigment epithelium; Spectral; Tomography, optical coherence; Vitelliform macular dystrophy

Best vitelliform macular dystrophy (BVMD) is a rare autosomal dominant retinal disease with highly variable phenotypic expression [1,2]. The disease-causing gene BEST1 is located on chromosome 11q13 and encodes bestrophin-1 [3-5], a multifunctional transmembrane protein that is localized in the basolateral plasma membrane and intracellular space of retinal pigment epithelial (RPE) cells [6-9]. Whether bestrophin functions directly as a $\mathrm{Ca}^{2+}$-sensitive chloride channel or as a regulator of ion transport, compelling evidence suggests that it is involved in the light peak conductance registered on electrooculography (EOG) [10-15]. Clinical suspicion of BVMD is based on the typical macular findings initially described by Best, who recognized an evolving spectrum of vitelliform macular lesions with advancing age [16] often in the setting of a positive family history. The diagnosis is confirmed by an abnormal light rise on the EOG [17-19], or by the identification of BEST1 gene mutation [20].

The definite diagnosis of BVMD, however, may be complicated. From a clinical perspective, atypical presentations such as multifocal vitelliform lesions [21-26], as well as the occurrence of choroidal neovascularization in various stages of the disease may confound the diagnosis at first [24,27-36]. A reliable EOG test is dependent on patients' full cooperation, which is a challenge in the pediatric population. Finally, while genetic tests to identify the BEST1 gene mutation are routinely performed in some referral centers, the access to this high-cost analysis remains restricted in many countries, and the phenotypicgenotypic correlation has been inconsistent so far since specific mutations were associated with a wide range of clinical manifestations [1,21-23,37,38].

Therefore, the identification of additional phenotypic findings that would facilitate the diagnosis of BVMD is highly desirable. In this manuscript, we describe the morphological macular features in patients with BVMD undergoing multimodal fundus imaging and make 
comparisons with those of normal age-matched subjects, with particular interest in findings obtained on Fourier-domain optical coherence tomography (Fd-OCT) guided by blue-light fundus autofluorescence (FAF) and by near-infrared reflectance (NIR).

\section{Materials and methods}

Patients with BVMD identified through a review of medical records between January 2003 and September 2009 at the Macular Imaging and Treatment Division of the Hospital de Olhos de Araraquara and at the Department of Ophthalmology of Columbia University were invited to participate in the study. First-order relatives were contacted when possible and invited to participate. The study protocol followed the statements of the Declaration of Helsinki and was approved by local Institutional Review Boards (\#10121/2008 and \#AAAB6560, respectively). Written informed consent was obtained for all participants prior to their inclusion in the study.

All participants underwent a complete ophthalmologic examination including best-corrected visual acuity (BCVA) utilizing early treatment diabetic retinopathy study (ETDRS) charts, slit-lamp biomicroscopy, and applanation tonometry. For illiterate participants, BCVA was measured using Snellen "E" charts. EOG testing was performed followed by a dilated fundus examination [39]. Due to local unavailability of genetic analysis, the diagnosis of BVMD was based on the presence of a positive family history with at least one affected first-order relative and an abnormally low to absent EOG light rise (Arden ratio <1.5) [39] with or without abnormal funduscopic changes [2,9,17-20]. For those patients meeting the diagnostic criteria, color, red-free, NIR, and FAF imaging were performed. The FAF was documented with an excitation wavelength of $488 \mathrm{~nm}$ and the emitted light detected above a 500-nm barrier filter, using the "high-resolution" mode with the "automatic real time" (ART) mean module set at 25 frames and "normalized" function activated. Stereoscopic fluorescein and indocyanine green (ICG) angiography were also performed if choroidal neovascular proliferation associated with the vitelliform lesion was suspected based upon prior investigations.

For the purpose of the current study, BVMD lesions were staged essentially according to the clinical classification proposed by Gass [2]. A deposit of yellowish material in the center of the macula resembling an egg yolk characterizes the "vitelliform" stage. Initial disruption of the vitelliform lesion with gravitational displacement of the material refers to the "pseudohypopyon" stage. Further disruption with remnants of yellowish material irregularly distributed and associated with RPE mobilization characterizes the "scrambled-egg" or "vitelliruptive" stage. Eventual absorption of the material and subretinal fluid leaving a flat area of RPE changes defines the "atrophic" stage.

Age-matched subjects were enrolled as controls. For inclusion, control subjects had to have a LogMAR ETDRS BCVA (converted to Snellen) equal or better than 20/25, a spherical equivalent refractive error of no greater than \pm 1.50 diopter, intraocular pressure of less than $21 \mathrm{mmHg}$ and no prior history or clinical evidence of retinal or optic nerve disease.

All participants underwent Fd-OCT imaging on a commercially available device (Spectralis ${ }^{\circledR}$ HRA+OCT; Heidelberg Engineering Inc., Heidelberg, Germany). Two types of scan acquisition protocols were used. The first protocol utilized the built-in scan acquisition function termed "volume" to acquire three-dimensional Fd-OCT data in a raster pattern consisting of $49 \mathrm{~B}$-scans covering a $20^{\circ} \times 20^{\circ}$ fundus area (equivalent to $\sim 5.6 \times 5.6 \mathrm{~mm}$ ). The second protocol utilized the built-in scan acquisition function termed "section" to acquire several B-scans covering a linear $30^{\circ}$ fundus area (equivalent to $\sim 9 \mathrm{~mm}$ ) in both the horizontal and vertical orientations which were positioned under discretion of the examiners 
during manual raster scanning of the entire macular area according to the reference fundus images (such as FAF, for example). The "high-resolution" mode with the ART mean module set at 25 frames was utilized for both acquisition protocols. The Fd-OCT data were qualitatively assessed in all participants, and simultaneous acquisition of Fd-OCT scans and multimodal reference images allowed point-to-point correlation of fundus features.

\section{Results}

Fourteen eyes of seven patients with BVMD and 14 eyes of seven age-matched controls were included. The mean age of enrolled subjects was 33 years (range 5 to 62 years) for patients and 34 years (range 7 to 66 years) for controls, and they were all male. The median LogMAR EDTRS BCVA (converted to Snellen) of patients and controls was 20/50 (range, $20 / 20$ to 20/320) and 20/20 (range, 20/20 to 20/25), respectively (Table 1). According to the clinical staging adopted in the current study, one patient had a "vitelliform" lesion in the left eye and a "vitelliruptive" lesion in the right eye, one patient had "pseudohypopyon" lesions in both eyes, three patients had "vitelliruptive" lesions in both eyes, and one patient had a "vitelliruptive" lesion in the left eye and an "atrophic" lesion in the right eye. The remaining patient had no macular lesion detectable on clinical examination.

\section{"Subclinical" stage}

In one patient (age 5 years) with a positive family history of BVMD (his father, one firstorder uncle, and a first-order cousin have BVMD with symptomatic fundus lesions), an abnormally low light rise result was observed in both eyes (Arden ratio equal to 1.19 [right eye], and to 1.17 [left eye]). Although the clinical evaluation was otherwise unremarkable, Fd-OCT imaging demonstrated a prominent highly reflective layer (HRL) between the outer-HRL (corresponding to the RPE/Bruch's membrane reflective complex) and the innerHRL (corresponding to the junction of the inner and outer segments of the photoreceptors, or IS/OS junction) in both eyes (Fig. 1). This reflective layer at the level of the photoreceptors' outer segments (OS) was revealed with the advent of high-resolution OCT [40], and for the purpose of this study is referred to as the middle-HRL as its precise histological correlation remains a matter of debate. When compared to the Fd-OCT images of the age-matched control eyes, the middle-HRL in this patient was abnormally thickened throughout the macula, particularly at the fovea. The total retinal thickness at the center of the fovea was not increased due to concomitant thinning of the outer nuclear layer (ONL). Hyporeflective spaces corresponding to fluid were not observed on Fd-OCT evaluation. No differences between patient and control were noted on NIR images. The FAF was apparently unremarkable, but high-quality documentation was not achieved due to poor cooperation (Fig. 1). Given that no follow-up is presented and no genetic analysis was performed as well as the fact that BVMD is an autosomal dominant disease with incomplete penetrance and variable expressivity, the term "subclinical" was preferred to the terms "previtelliform" or "carrier" to stage this particular lesion.

\section{"Vitelliform" stage}

In one patient (age 6 years), an elevated yellow round macular lesion measuring approximately $3 / 4$ disc diameter across was found in the left eye. On Fd-OCT imaging, the foveal retina was elevated due to a homogeneous hyper-reflective material located just above the outer-HRL (RPE/Bruch complex). The middle-HRL was elevated at the margins of the hyper-reflective material and followed the contour of the lesion with areas of thickening and fragmentation above the hyper-reflective material. The ONL appeared thinned above the hyper-reflective material due to heterogeneous hyper-reflective signals that are not commonly observed at this layer. Hyporeflective spaces corresponding to fluid were not observed on Fd-OCT evaluation. The NIR imaging clearly delineated the margins 
of the vitelliform lesion. The hyper-reflective material observed between the outer-HRL and middle-HRL (thus localized presumably between the OS and RPE layers) presented increased fluorescence on FAF (Fig. 2). Combined Fd-OCT and FAF evaluation revealed that the ONL was somewhat thinned beyond the clinically evident margins of the lesion along its entire contour. In addition, thickening of the middle-HRL, identical to that observed in the child with subclinical stage above described (Fig. 1), was also documented in regions of normal fluorescence on FAF close to the vitelliform macular lesion (Fig. 2).

\section{"Pseudohypopyon" stage}

In one patient (age 19 years), an elevated oval macular lesion measuring approximately three disc diameters across was observed in both eyes. A yellowish material located more prominently at the inferior aspect of the lesion was evident. In the center of the macula, FdOCT imaging showed separation of the neurosensory retina from the outer-HRL (RPE/ Bruch complex) by an optically clear space and clumps of homogeneous hyper-reflective material overlying the RPE inferiorly displaced (Fig. 3). In the elevated neurosensory retina, the ONL was thinned when compared to the control eyes and presented focal regions of heterogeneous reflectivity. The inner-HRL (IS/OS junction) exhibited focal areas of disruption superiorly and nasally to the fovea in both eyes. The elevated middle-HRL (photoreceptors' OS) was irregularly thickened. The outer-HRL (RPE/Bruch complex) was relatively preserved beneath the elevated neurosensory retina, with small hyper-reflective mounds at this level. On FAF, increased autofluorescence from the hyper-reflective material overlying the RPE was observed from the inferior aspect of the lesion and, to a lesser degree, from the margins of the lesion. Fundus NIR clearly delineated the retinal elevation. Of note, in the macular region above the vitelliform lesion with normal fluorescence on FAF, the Fd-OCT imaging revealed a distinctively thickened middle-HRL when compared with corresponding areas in the control eyes.

\section{"Vitelliruptive" stage}

In eight eyes of five patients (age 6-62 years) elevated macular lesions measuring from 1 $1 / 2$ to 3 disc diameters across were observed. Variable amounts of yellowish subretinal material were dispersed within and at the margins of the macular lesions in all eight eyes; in five of these, a distinct grayish subretinal tissue was evident at the center of the lesions. In all eight eyes, Fd-OCT imaging showed the neurosensory retina separated from the outerHRL (RPE/Bruch complex) centrally by an optically clear space. In the elevated neurosensory retina, there was marked thinning of the ONL and a variable degree of alterations of the middle- and inner- HRLs (photoreceptors' OS and IS/OS junction, respectively) when compared to control eyes. In one patient (age 6 years), the photoreceptors' OS were irregularly elongated. In the remaining patients (age 37-62 years), regions of hyper-reflectivity from the IS/OS junction and abnormal elongation of the OS were interspersed with areas of complete absence of photoreceptors' signal.

The subretinal grayish tissue at the center of the dome-shaped lesion corresponded to hyperreflective mounds at the level of outer-HRL (RPE/Bruch complex). Two somewhat distinct hyper-reflective mounds were identified on integrated evaluation of multimodal fundus imaging. The first mound was associated with relative shadowing of the underlying choroid signals on Fd-OCT, had no characteristic findings on FAF and was poorly delineated on NIR (Fig. 4). The second hyper-reflective mound was invariably associated with secondary retinal changes; the outer retinal layers were indistinguishable and generally collapsed over the mound. In addition, unlike the first, this mound was associated with relative hyperreflectivity of the underlying choroid, and it was clearly delineated on FAF and NIR imaging (Fig. 4). The yellowish subretinal material observed clinically tended to accumulate at the margins of the macular lesion and presented characteristics similar to the yellowish 
material also observed in eyes with vitelliform and pseudohypopyon stages, appearing as a homogeneous hyper-reflective material just above the outer-HRL (RPE/Bruch complex) on Fd-OCT and exhibiting increased fluorescence on FAF. The Fd-OCT imaging beyond the dome-shaped lesion demonstrated diffuse thickening of the middle-HRL throughout the macular area when compared with control eyes.

\section{“Atrophic" stage}

In one eye of one patient (age 62 years), we observed an irregular oval area of RPE mobilization of approximately $21 / 2$ disc diameters across encompassing the macula with no apparent retinal elevation. Within the lesion area, Fd-OCT imaging revealed overall thinning of the neurosensory retina with a shallow separation from the outer-HRL (RPE/Bruch complex) by an optically clear space. The photoreceptors' reflective complex (OS/innerHRL/IS) was practically absent in the fovea, and the ONL was thinned throughout the macula when compared to the control eye. The outer-HRL (RPE/Bruch complex) was relatively preserved, and one isolated hyper-reflective mound with underlying choroidal shadowing was observed. A mild stippled fluorescence on FAF was observed throughout the macula. Fundus NIR was not helpful in delineating the extension of the macular lesion. Evaluation of the macular area around the lesion demonstrated that the middle-HRL was more evident and diffusely thicker throughout the macula when compared to control eyes.

\section{Discussion}

The pathogenesis of BVMD has been a matter of speculation since the disorder was first described. Since the encoded protein of BEST1 is expressed in the RPE, the primary dysfunctional site of BVMD apparently lies within the RPE cells [3,6,41]. In addition to the proposed mechanisms involving ionic conductance in the RPE cell, bestrophin activity possibly influences intracellular processes such as phagocytosis and lysosomal function [9,42-44] as well as vascular endothelium growth factor production by the RPE [45]. The few histopathological studies reported to date are conflicting in fundamental issues such as whether the primary tissue damage evolves with RPE or neurosensory retinal changes $[28,29,41,46-48]$. Interpretations of pathophysiologic mechanisms based upon OCT findings in BVMD have also lacked agreement among investigators [19,20,49-54]. Some authors concluded that the initial morphological events in patients with BVMD also occur at the level of the RPE monolayer [49]. In the current study, no obvious alteration could be demonstrated on Fd-OCT specifically at the reflective layer corresponding to the dysfunctional RPE monolayer in the macula of eyes with subclinical or vitelliform lesions. Conversely, the reflective layer (middle-HRL) corresponding presumably to photoreceptors' OS was apparently thickened throughout the macula based on our interpretation (Fig. 1). Our impression was that the middle-HRL was also apparently thickened in macular regions with otherwise normal fluorescence on FAF (Fig. 2).

In patients with BVMD, Querques et al. recently demonstrated the presence of "previtelliform" lesions that were characterized by a "thicker and more reflective appearance of the layer between the RPE and the photoreceptor IS/OS interface in the central region, compared with the normal macula" [54]. These authors correlated this thickened layer to the Verhoeff's membrane [54,55]. Additionally, in the previtelliform stage, they reported a "normal appearance of all major intraretinal layers from ILM to ELM, as well as a normalappearing RPE and IS/OS interface, were found in two eyes (50\%); focal disruption of IS/ OS interface and of the layer between the RPE and the IS/OS interface was observed in another two eyes (50\%)" [54]. In the current study, we also documented a thickened layer at the outer retina in both eyes of a 5-year-old child with an unremarkable clinical examination. However, unlike Querques et al., we believe that this particular finding occurs at the level of the middle-HRL, which corresponds to photoreceptors' OS reflective layer and not to 
"Verhoeff's membrane" [54], which corresponds to junctional complexes along the lateral cell membranes of the RPE that on light microscopy produce the effect of a continuous membrane at the RPE level [56]. Moreover, the middle-HRL was apparently thickened throughout the macula of both eyes with secondary ONL thinning observed centrally (Fig. 1). Based on the multimodal imaging documentation we obtained, these particular findings were observed in regions of normal fluorescence on FAF with no evidence of subretinal fluid accumulation (Figs. 1 and 2).

Histopathological studies of vitelliruptive lesions reported variable degrees of photoreceptors' cell damage with descriptions ranging from "total atrophy" to "IS of surviving photoreceptors shortened and tipped by only small bits of OS material". Notable attenuation of the ONL was a constant finding in these studies $[28,29,46]$. These histopathologic observations are in agreement with our findings. We demonstrated a variable degree of photoreceptor damage on Fd-OCT, which ranged from the absence of the entire photoreceptors' reflective layer with adjacent ONL thinning in an eye at the atrophic stage to a relatively well-preserved IS/OS junction associated with elongated OS and reduced ONL in eyes at earlier disease stages. Coupled with the clinical results (BCVA measurements) and observations from FAF documentation, we believe that the hyperreflective signals at the level of the OS/inner-HRL/IS reflective layer observed on Fd-OCT may correspond mainly to segments of "surviving photoreceptors' cells". In the current study, as long as reflective signals from the OS/inner-HRL/IS layer were present in the foveolar region, the BCVA was as good as 20/25 in eyes with vitelliform or pseudohypopyon lesions, and 20/32 in an eye with a vitelliruptive lesion. In contrast, BCVA was decreased to 20/320 in an eye with atrophic lesion and absence of the entire OS/innerHRL/IS reflective layer within the foveola (Fig. 5).

This study has obvious limitations, such as the small number of patients and the absence of a normative database for the thickness of the different photoreceptors' reflective layers at the present moment, which preclude any definitive conclusion to be made. Genetic analysis looking for possible genotype-phenotype correlation regarding the Fd-OCT findings herein suggested as well as for potential differences in Fd-OCT features between subclinical stages and carrier status should also be considered in future studies. However, the concordance between our multimodal imaging findings and previous histopathological [29] and functional [57] studies in BVMD patients apparently support our observations. Finally, as far as we are aware, this is the first study to suggest in vivo morphological changes in the photoreceptors' OS reflective layer diffusely throughout the macula (and not only in the lesion area), as well as the early involvement of the ONL centrally in the macula. These impressions, if confirmed in future studies utilizing multimodal fundus imaging, may ultimately have implications for early diagnosis in patients with BVMD.

\section{References}

1. Maloney WF, Robertson DM, Duboff SM. Hereditary vitelliform macular degeneration: variable fundus findings within a single pedigree. Arch Ophthalmol 1977;95:979-983. [PubMed: 869756]

2. Gass, JDM. Stereoscopic atlas of macular diseases, diagnosis and treatment. Mosby: St. Louis; 1997.

3. Stone EM, Nichols BE, Streb LM, Kimura AE, Sheffield VC. Genetic linkage of vitelliform macular degeneration (Best's disease) to chromosome 11q13. Nat Genet 1992;1:246-250. [PubMed: 1302019]

4. Petrukhin K, Koisti MJ, Bakall B, Li W, Xie G, Marknell T, Sandgren O, Forsman K, Holmgren G, Andreasson S, Vujic M, Bergen AAB, McGarty-Dugan V, Figueroa D, Austin CP, Metzker ML, Caskey CT, Wadelius C. Identification of the gene responsible for Best macular dystrophy. Nat Genet 1998;19:241-247. [PubMed: 9662395] 
5. Marquardt A, Stohr H, Passmore LA, Kramer F, Rivera A, Weber BH. Mutations in a novel gene, VMD2, encoding a protein of unknown properties cause juvenile-onset vitelliform macular dystrophy (Best disease). Hum Mol Genet 1998;7:1517-1525. [PubMed: 9700209]

6. Marmorstein AD, Marmorstein LY, Rayborn M, Wang X, Hollyfield JG, Petrukhin K. Bestrophin, the product of the Best vitelliform macular dystrophy gene (VMD2), localizes to the basolateral plasma membrane of the retinal pigment epithelium. Proc Natl Acad Sci USA 2000;97:1275812763. [PubMed: 11050159]

7. Bakall B, McLaughlin P, Stanton JB, Zhang Y, Hartzell HC, Marmorstein LY, Marmorstein AD. Bestrophin-2 is involved in the generation of intraocular pressure. Invest Ophthalmol Vis Sci 2008;49:1563-1570. [PubMed: 18385076]

8. Yu K, Qu Z, Cui Y, Hartzell HC. Chloride channel activity of bestrophin mutants associated with mild or late-onset macular degeneration. Invest Ophthalmol Vis Sci 2007;48:4694-4705. [PubMed: 17898294]

9. Boon CJF, Klevering BJ, Leroy BP, Hoyng CB, Keunen JEE, den Hollander AI. The spectrum of ocular phenotypes caused by mutations in the BEST1 gene. Prog Retin Eye Res 2009;28:187-205. [PubMed: 19375515]

10. Tsunenari T, Sun H, Williams J, Cahill H, Smallwood P, Yau KW, Nathans J. Structure-function analysis of the bestrophin family of anion channels. J Biol Chem 2003;278:41114-41125. [PubMed: 12907679]

11. Marmorstein AD, Kinnick TR. Focus on molecules: Bestrophin (Best-1). Exp Eye Res 2007;85:423-424. [PubMed: 16720022]

12. Sun H, Tsunenari T, Yau KW, Nathans J. The vitelliform macular dystrophy protein defines a new family of chloride channels. Proc Natl Acad Sci USA 2002;99:4008-4013. [PubMed: 11904445]

13. Yu K, Cui Y, Hartzell HC. The bestrophin mutation A243V, linked to adult-onset vitelliform macular dystrophy, impairs its chloride channel function. Invest Ophthalmol Vis Sci 2006;47:4956-4961. [PubMed: 17065513]

14. Hartzell HC, Qu Z, Yu K, Xiao Q, Chien LT. Molecular physiology of bestrophins: multifunctional membrane proteins linked to Best disease and other retinopathies. Physiol Rev 2008;88:639-672. [PubMed: 18391176]

15. Marmorstein AD, Cross HE, Peachey NS. Functional roles of bestrophins in ocular epithelia. Prog Retin Eye Res 2009;28:206-226. [PubMed: 19398034]

16. Best F. Über eine hereditäre Makulaaffektion. Z F Augenheilk 1905;13:199-212.

17. Deutman AF. Electro-oculography in families with vitelliform dystrophy of the fovea. Detection of the carrier state. Arch Ophthalmol 1969;81:305-316. [PubMed: 5774285]

18. Cross HE, Bard L. Electro-oculography in Best macular dystrophy. Am J Ophthalmol 1974;77:4650. [PubMed: 4824173]

19. Glybina IV, Frank RN. Localization of multifocal electro-retinogram abnormalities to the lesion site: findings in a family with Best disease. Arch Ophthalmol 2006;124:1593-1600. [PubMed: 17102007]

20. Boon CJF, Theelen T, Hoefsloot EH, van Schooneveld MJ, Keunen JEE, Cremers FPM, Klevering BJ, Hoyng CB. Clinical and molecular genetic analysis of Best vitelliform macular dystrophy. Retina 2009;29:835-847. [PubMed: 19357557]

21. Renner AB, Tillack H, Kraus H, Kramer F, Mohr N, Weber BHF, Foerster MH, Kellner U. Late onset is common in Best macular dystrophy associated with VMD2 gene mutations. Ophthalmology 2005;112:586-592. [PubMed: 15808248]

22. Wabbels B, Preising MN, Kretschmann U, Demmler A, Lorenz B. Genotype-phenotype correlation and longitudinal course in ten families with Best vitelliform macular dystrophy. Graefes Arch Clin Exp Ophthalmol 2006;244:1453-1466. [PubMed: 16612637]

23. Boon CJ, Klevering BJ, den Hollander AI, Zonneveld MN, Theelen T, Cremers FPM, Hoyng CB. Clinical and genetic heterogeneity in multifocal vitelliform dystrophy. Arch Ophthalmol 2007;125:1100-1106. [PubMed: 17698758]

24. Mohler CW, Fine SL. Long-term evaluation of patients with Best vitelliform dystrophy. Ophthalmology 1981;88:688-692. [PubMed: 7267039] 
25. Boon CJ, Klevering JB, Keunen JE, Hoyng CB, Theelen T. Fundus autofluorescence imaging of retinal dystrophies. Vis Res 2008;48:2569-2577. [PubMed: 18289629]

26. Querques G, Regenbogen M, Soubrane G, Souied EH. High-resolution spectral domain optical coherence tomography findings in multifocal vitelliform macular dystrophy. Surv Ophthalmol 2009;54:311-316. [PubMed: 19298908]

27. Noble KG, Scher BM, Carr RE. Polymorphous presentations in vitelliform macular dystrophy: subretinal neovascularization and central choroidal atrophy. Br J Ophthalmol 1978;62:561-570. [PubMed: 687557]

28. Frangieh GT, Green WR, Fine SL. A histopathologic study of Best macular dystrophy. Arch Ophthalmol 1982;100:1115-1121. [PubMed: 7092655]

29. O'Gorman S, Flaherty WA, Fishman GA, Berson EL. Histopathologic findings in Best vitelliform macular dystrophy. Arch Ophthalmol 1988;106:1261-1268. [PubMed: 3415551]

30. Blodi CF, Stone EM. Best vitelliform dystrophy. Ophthalmic Paediatr Genet 1990;11:49-59. [PubMed: 2190134]

31. Fishman GA, Baca W, Alexander KR, Derlacki DJ, Glenn AM, Viana M. Visual acuity in patients with Best vitelliform macular dystrophy. Ophthalmology 1993;100:1665-1670. [PubMed: 8233392]

32. Chung MM, Oh KT, Streb LM, Kimura AE, Stone EM. Visual outcome following subretinal hemorrhage in Best disease. Retina 2001;21:575-580. [PubMed: 11756879]

33. Andrade RE, Farah ME, Cardillo JA, Hofling-Lima AL, Uno F, Costa RA. Optical coherence tomography in choroidal neovascular membrane associated with Best vitelliform dystrophy. Acta Ophthalmol Scand 2002;80:216-218. [PubMed: 11952492]

34. Andrade RE, Farah ME, Costa RA. Photodynamic therapy with verteporfin for subfoveal choroidal neovascularization in Best disease. Am J Ophthalmol 2003;136:1179-1181. [PubMed: 14644242]

35. Leu J, Schrage NF, Degenring RF. Choroidal neovascularization secondary to Best disease in a 13year-old boy treated by intravitreal bevacizumab. Graefes Arch Clin Exp Ophthalmol 2007;245:1723-1725. [PubMed: 17605026]

36. Querques G, Bocco MC, Soubrane G, Souied EH. Intravitreal ranibizumab (Lucentis) for choroidal neovascularization associated with vitelliform macular dystrophy. Acta Ophthalmol 2008;86:694695. [PubMed: 18752521]

37. Bakall B, Marknell T, Ingvast S, Koisti MJ, Sandgren O, Li W, Bergen AAB, Andreasson S, Rosenberg T, Petrukhin K, Wadelius C. The mutation spectrum of the bestrophin proteinfunctional implications. Hum Genet 1999;104:383-389. [PubMed: 10394929]

38. Kramer F, White K, Pauleikhoff D, Gehrig A, Passmore L, Rivera A, Rudolph G, Kellner U, Andrassi M, Lorenz B, Rohrschneider K, Blankenagel A, Jurklies B, Schilling H, Schutt F, Holz FG, Weber BH. Mutations in the VMD2 gene are associated with juvenile-onset vitelliform macular dystrophy (Best disease) and adult-onset vitelliform macular dystrophy but not agerelated macular degeneration. Eur J Hum Genet 2000;8:286-292. [PubMed: 10854112]

39. Brown M, Marmor M, Vaegan ZE, Brigell M, Bach M. ISCEV. ISCEV standard for clinical electro-oculography (EOG). Doc Ophthalmol 2006;113:205-212. [PubMed: 17109157]

40. Srinivasan VJ, Monson BK, Wojtkowski M, Bilonick RA, Gorczynska I, Chen R, Duker JS, Schuman JS, Fujimoto JG. Characterization of outer retinal morphology with high-speed, ultrahigh-resolution optical coherence tomography. Invest Ophthalmol Vis Sci 2008;49:15711579. [PubMed: 18385077]

41. Mullins RF, Kuehn MH, Faidley EA, Syed NA, Stone EM. Differential macular and peripheral expression of bestrophin in human eyes and its implication for Best disease. Invest Ophthalmol Vis Sci 2007;48:3372-3380. [PubMed: 17591911]

42. Deguchi J, Yamamoto A, Yoshimori T, Sugasawa K, Moriyama Y, Futai M, Suzuki T, Kato K, Uyama M, Tashiro Y. Acidification of phagosomes and degradation of rod outer segments in rat retinal pigment epithelium. Invest Ophthalmol Vis Sci 1994;35:568-579. [PubMed: 8113008]

43. Jentsch TJ. Chloride and the endosomal-lysosomal pathway: emerging roles of CLC chloride transporters. J Physiol 2007;578:633-640. [PubMed: 17110406] 
44. Karl MO, Kroeger W, Wimmers S, Milenkovic VM, Valtink M, Engelmann K, Strauss O. Endogenous Gas6 and $\mathrm{Ca}^{2+}$-channel activation modulate phagocytosis by retinal pigment epithelium. Cell Signal 2008;20:1159-1168. [PubMed: 18395422]

45. Rosenthal R, Heimann H, Agostini H, Martin G, Hansen LL, Strauss O. Ca2+ channels in retinal pigment epithelial cells regulate vascular endothelial growth factor secretion rates in health and disease. Mol Vis 2007;13:443-456. [PubMed: 17417605]

46. Weingeist T, Kobrin J, Watzke R. Histopathology of Best macular dystrophy. Arch Ophthalmol 1982;100:1108-1114. [PubMed: 7092654]

47. Mullins RF, Oh KT, Heffron E, Hageman G, Stone EM. Late development of vitelliform lesions and flecks in a patient with Best disease. Arch Ophthalmol 2005;123:1588-1594. [PubMed: 16286623]

48. Bakall B, Radu RA, Stanton JB, Burke JM, McKay BS, Wadelius C, Mullins RF, Stone EM, Travis GH, Marmorstein AD. Enhanced accumulation of A2E in individuals homozygous or heterozygous for mutations in BEST1 (VMD2). Exp Eye Res 2007;85:34-43. [PubMed: 17477921]

49. Pianta MJ, Aleman TS, Cideciyan AV, Sunness JS, Li Y, Campochiaro BA, Campochiaro PA, Zack DJ, Stone EM, Jacobson SG. In vivo micropathology of Best macular dystrophy with optical coherence tomography. Exp Eye Res 2003;76:203-211. [PubMed: 12565808]

50. Men G, Batioglu F, Ozkan SS, Huban A, Ozdamar Y, Aslan O. Best vitelliform macular dystrophy with pseudohypopyon: an optical coherence tomography study. Am J Ophthalmol 2004;137:963965. [PubMed: 15126177]

51. Vedantham V, Ramasamy K. Optical coherence tomography in Best disease: an observational case report. Am J Ophthalmol 2005;139:351-353. [PubMed: 15734003]

52. Spaide RF, Noble K, Morgan A, Freund KB. Vitelliform macular dystrophy. Ophthalmology 2006;113:1392-1400. [PubMed: 16877078]

53. Spaide RF. Autofluorescence from the outer retina and subretinal space. Hypothesis and review. Retina 2008;28:5-35. [PubMed: 18185134]

54. Querques G, Regenbogen M, Quijano C, Delphin N, Soubrane G, Souied EH. High-definition optical coherence tomography features in vitelliform macular dystrophy. Am J Ophthalmol 2008;146:501-507. [PubMed: 18619572]

55. Zawadzki RJ, Jones SM, Olivier SS, Zhao M, Bower BA, Izatt JA, Choi S, Laut S, Werner JS. Adaptive-optics optical coherence tomography for high-resolution and high-speed 3D retinal in vivo imaging. Opt Express 2005;13:8532-8546. [PubMed: 19096728]

56. Verhoeff FH. A hitherto undescribed membrane of the eye and its significance. Boston Med Surg J 1903;149:456.

57. Maia-Lopes S, Silva ED, Reis A, Silva MF, Mateus C, Castelo-Branco M. Retinal function in Best macular dystrophy: relationship between electrophysiological, pychophysical, and structural measures of damage. Invest Ophthalmol Vis Sci 2008;49:5553-5560. [PubMed: 18775865] 

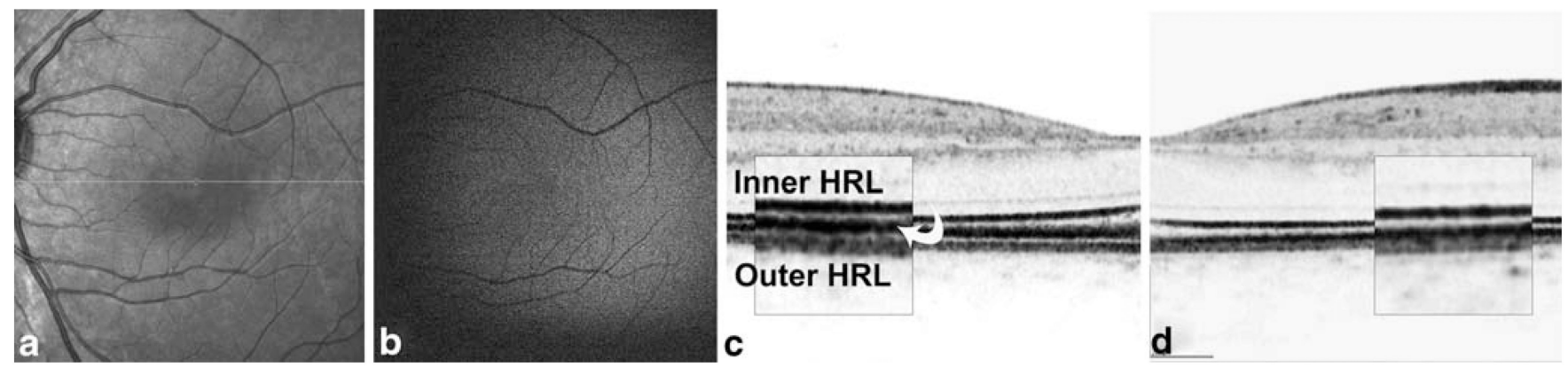

Fig. 1.

Near-infrared reflectance (a) and blue-light fundus autofluorescence (b) imaging of a 5year-old patient in the "subclinical" stage of Best vitelliform macular dystrophy (normal clinical and fundus photography evaluations; best-corrected visual acuity was 20/20). On Fourier-domain optical coherence tomography imaging $(\mathbf{c})$, the middle highly reflective layer (HRL) (photoreceptors' outer segments; curved arrow) between the inner-HRL (junction of photoreceptors' inner/outer segments) and outer-HRL (RPE/Bruch's membrane reflective complex) was abnormally distinct throughout the macula and thicker when compared with the age-matched control (d), with no difference in the total foveal height $(257 \mu \mathrm{m})($ bars $=200 \mu \mathrm{m})$ 

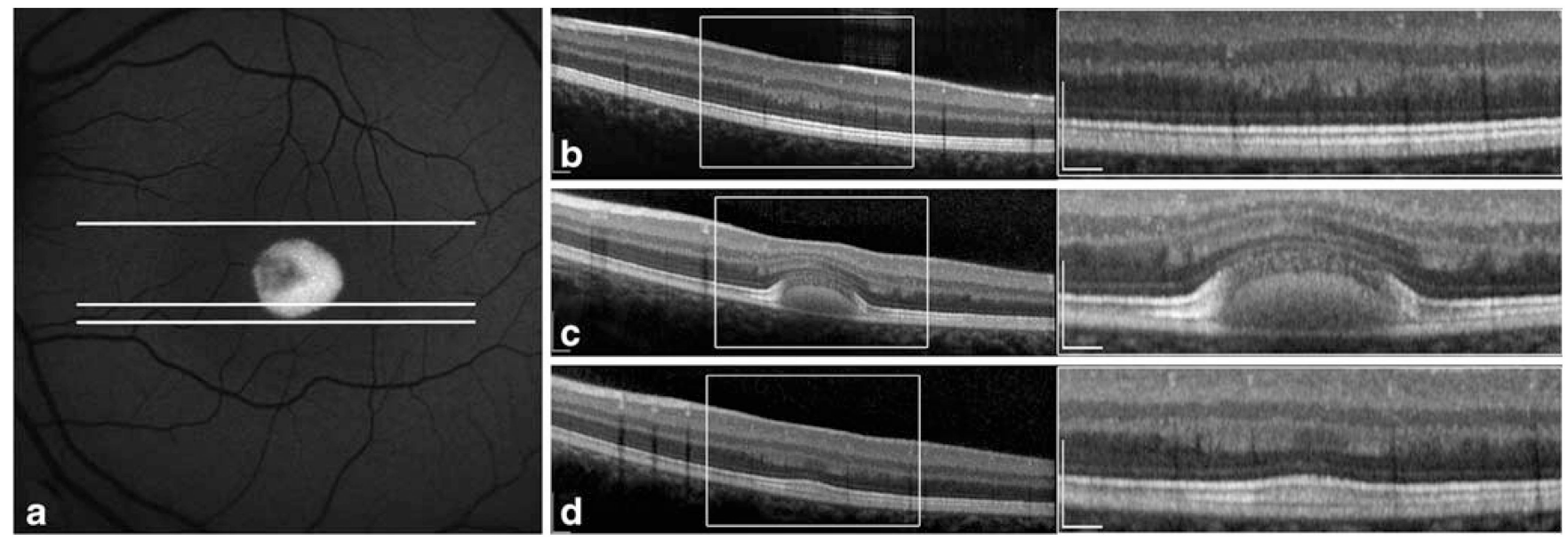

Fig. 2.

Blue-light fundus autofluorescence (FAF) (a) and Fourier-domain optical coherence tomography (Fd-OCT) imaging (horizontal B-scans, b, c, and d) in a 6-year-old patient with Best vitelliform macular dystrophy in the "vitelliform" stage. Increased autofluorescence on FAF was observed from the vitelliform lesion (a). The Fd-OCT imaging guided by simultaneous FAF revealed that the middle highly reflective layer (photoreceptors' outer segments) remained apparently thickened, even in areas with normal fluorescence on FAF. Right panel shows corresponding magnifications of the white rectangles 

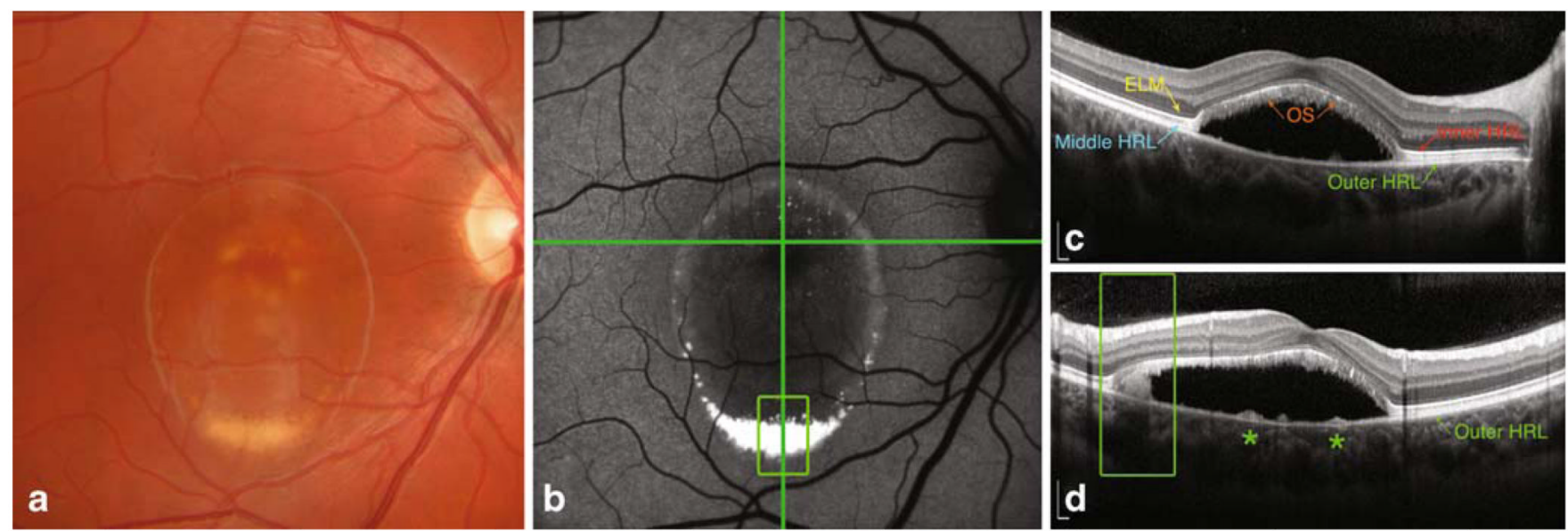

Fig. 3.

Color fundus photography (a), blue-light fundus autofluorescence (FAF) (b), and Fourierdomain optical coherence tomography (Fd-OCT) imaging (horizontal B-scan, c; vertical Bscan, d) of one patient with Best vitelliform macular dystrophy in the "pseudohypopyon" stage. In the elevated retina, the middle highly reflective layer corresponding to photoreceptors' outer segments was thickened and abnormally hyper-reflective, and the outer nuclear layer was thinned (c). The yellowish vitelliform material displaced inferiorly showed increased FAF (open square green box), and appeared as homogeneous clumps lying free over the outer-HRL (RPE/Bruch complex) on Fd-OCT (d). Subretinal tissue at the center of the macula was not evident on FAF, but was clearly documented on Fd-OCT as small hyper-reflective mounds at the level of the outer-HRL (asterisks, d) (bars $=200 \mu \mathrm{m})$ 

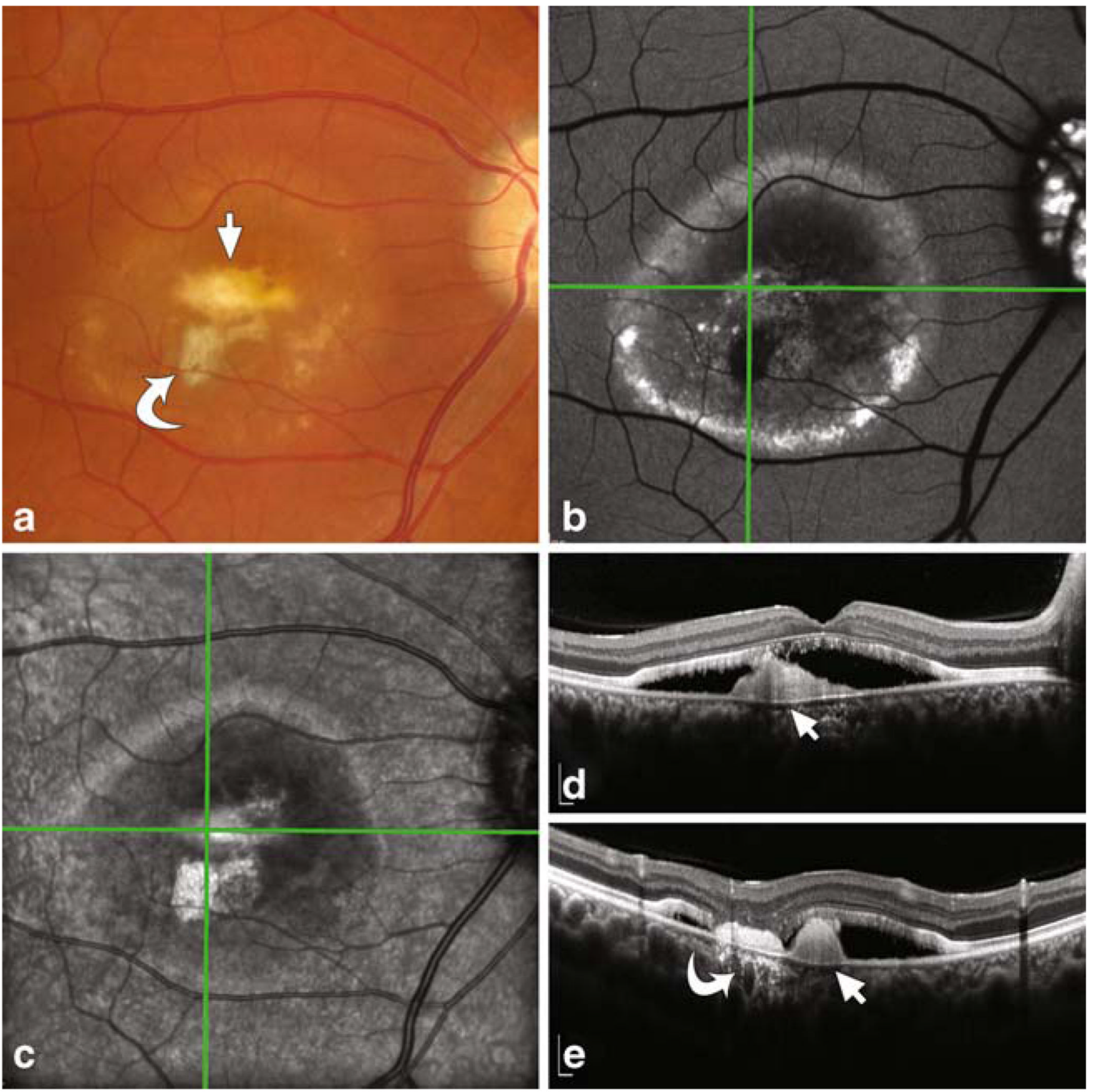

Fig. 4.

Color fundus photography (a), blue-light fundus autofluorescence (FAF) (b), near-infrared photography (NIR) (c), and Fourier-domain optical coherence tomography (Fd-OCT) imaging (horizontal scan, d; vertical scan, e) of one patient with Best vitelliform macular dystrophy in the "vitelliruptive" stage. The subretinal tissue at the center of the dome-shaped lesion (arrowhead and curved arrow in a) corresponded to hyper-reflective mounds at the level of outer-HRL (RPE/Bruch complex) with different characteristics. The first mound (arrowhead, $\mathbf{d}$ and $\mathbf{e}$ ) caused relative shadowing of the underlying choroid signals on FdOCT, while the second (curved arrow, e) was associated with overlying collapse of outer 
retinal layers and with relative hyper-reflectivity of the underlying choroid (bars $=200 \mu \mathrm{m}$ ). Note increased FAF from optic nerve drusen 

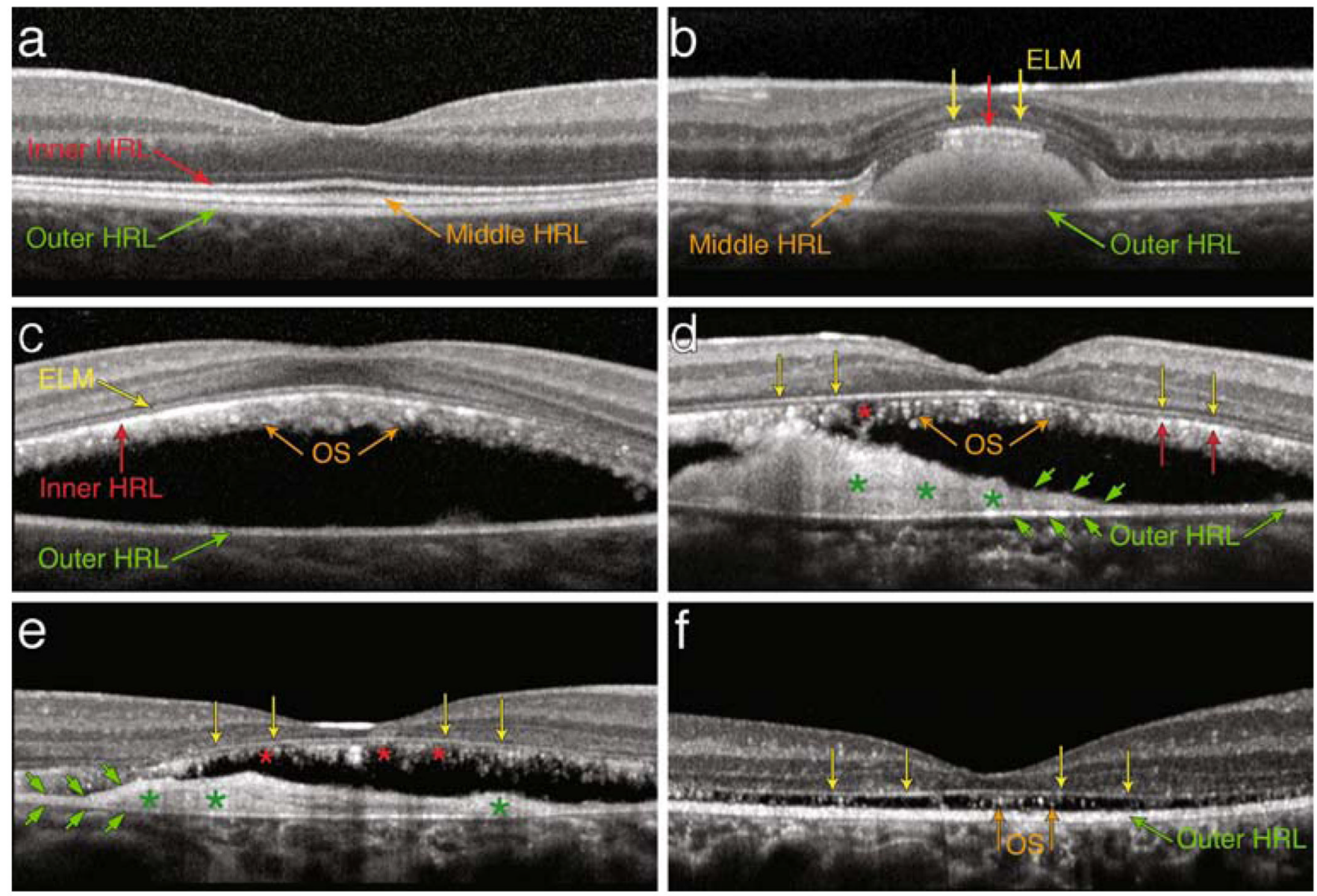

Fig. 5.

Fourier-domain optical coherence tomography (horizontal scans) from a spectrum of patients to illustrate the different stages of Best vitelliform macular dystrophy.

"Subclinical" stage (a): the middle highly reflective layer (HRL) correspondent to photoreceptors' outer segments (OS) is thicker throughout the macula; BCVAwas 20/20. "Vitelliform" stage (b): homogenous hyper-reflective material is observed between the outer-HRL (RPE/Bruch's membrane reflective complex) and the middle-HRL. Over the material, the photoreceptors' OS appears elongated, the outer nuclear layer (ONL) is thinned, and both the inner-HRL (IS/OS junction) and the external limiting membrane (ELM) are distinguishable (yellow arrow); BCVA was 20/32. "Pseudohypopyon" stage (c): the outer-HRL is fairly preserved (green arrow), subretinal fluid is observed (with inferior displacement of vitelliform material), photoreceptors' OS are elongated and irregular aligned (orange arrows), inner-HRL is regularly discernible (red arrow), and the ONL is thinned; BCVA was 20/32. "Vitelliruptive" stage (d and e): the OS become shortened and grossly misaligned (orange arrows), attenuation and fragmentation of the inner-HRL (red asterisks) are observed, and the ONL becomes so reduced that is no longer clearly discernible; split (green arrowheads) of the inner-HRL (green arrow) due to hyperreflective mounds (green asterisks) may be seen; BCVA was 20/100 in each eye. "Atrophic" stage (f): further loss of photoreceptors' cells occurs (orange arrows), and subretinal fluid may eventually reabsorb; in absence of hyper-reflective mounds, the outerHRL may be relatively well preserved (green arrow); BCVA was 20/320 


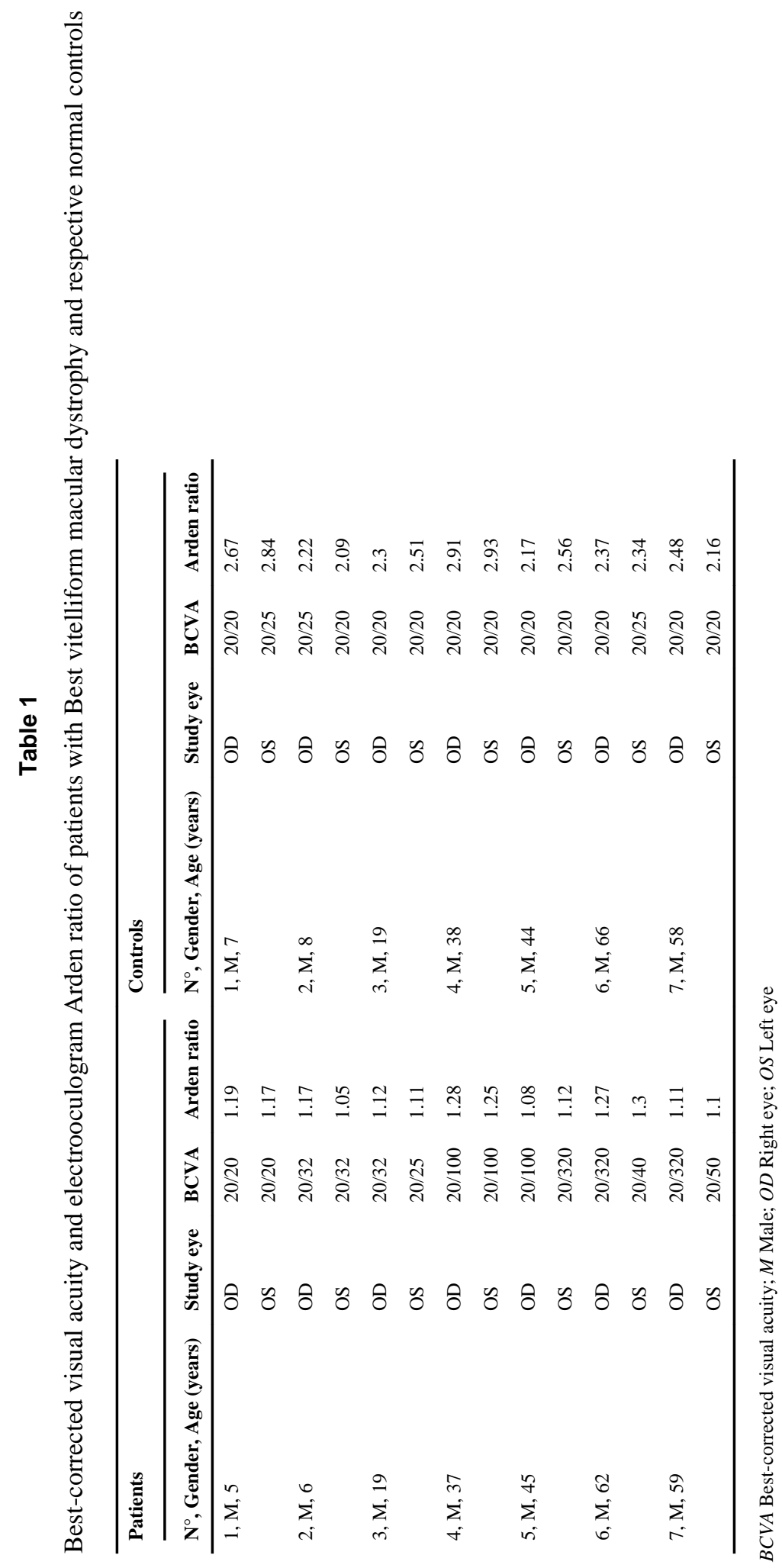

